\title{
Effects of balance training using a virtual-reality system in older fallers
}

\author{
This article was published in the following Dove Press journal: \\ Clinical Interventions in Aging \\ 27 February 2013 \\ Number of times this article has been viewed
}

\section{Gustavo Duque ${ }^{1,2}$ \\ Derek Boersma' \\ Griselda Loza-Diaz ${ }^{2}$ \\ Sanobar Hassan' \\ Hamlet Suarez ${ }^{3}$ \\ Dario Geisinger ${ }^{3}$ \\ Pushpa Suriyaarachchi' \\ Anita Sharma' \\ Oddom Demontiero',}

'Falls and Fractures Clinic, Department of Geriatric Medicine, Nepean Hospital, Penrith, NSW, Australia; ${ }^{2}$ Ageing Bone Research Program, Division of Geriatric Medicine, Sydney Medical School Nepean, The University of Sydney, Penrith, NSW, Australia; ${ }^{3}$ British Hospital, CLAEH School of Medicine, Montevideo, Uruguay
Correspondence: Gustavo Duque Ageing Bone Research Program, Division of Geriatric Medicine, Sydney Medical School Nepean, The University of Sydney, PO Box 63, Penrith, NSW 275I, Australia Tel +6I 247344278 Fax +6I $2473426 \mid 4$

Email gustavo.duque@sydney.edu.au

\begin{abstract}
Poor balance is considered a challenging risk factor for falls in older adults. Therefore, innovative interventions for balance improvement in this population are greatly needed. The aim of this study was to evaluate the effect of a new virtual-reality system (the Balance Rehabilitation Unit [BRU]) on balance, falls, and fear of falling in a population of community-dwelling older subjects with a known history of falls. In this study, 60 community-dwelling older subjects were recruited after being diagnosed with poor balance at the Falls and Fractures Clinic, Nepean Hospital (Penrith, NSW, Australia). Subjects were randomly assigned to either the BRU-training or control groups. Both groups received the usual falls prevention care. The BRU-training group attended balance training (two sessions/week for 6 weeks) using an established protocol. Change in balance parameters was assessed in the BRU-training group at the end of their 6-week training program. Both groups were assessed 9 months after their initial assessment (month 0). Adherence to the BRU-training program was $97 \%$. Balance parameters were significantly improved in the BRU-training group $(P<0.01)$. This effect was also associated with a significant reduction in falls and lower levels of fear of falling $(P<0.01)$. Some components of balance that were improved by BRU training showed a decline after 9 months post-training. In conclusion, BRU training is an effective and well-accepted intervention to improve balance, increase confidence, and prevent falls in the elderly.
\end{abstract}

Keywords: falls, balance, postural instability, virtual reality, elderly

\section{Introduction}

Falls in older persons are associated with fear of falling, lack of confidence, injuries, hospitalizations and, in some cases, death. ${ }^{1}$ Although broadly implemented, falls prevention programs are a major burden to health care budgets worldwide. This is due to the complexity of the secondary prevention programs and the limited adherence to them in both community and institutional settings. ${ }^{1,2}$

Older fallers suffering from balance problems constitute one of the most challenging types of patients in clinical practice. ${ }^{3}$ The only interventions that have demonstrated an effect on balance and falls prevention are vitamin D supplementation, Tai Chi, and exercise. ${ }^{4}$ A recent meta-analysis by Muir and Montero-Odasso ${ }^{5}$ reported that vitamin D supplementation has beneficial effects on strength and balance but not on gait. Another recent meta-analysis proposed Tai Chi programs as the most beneficial intervention to improve balance in older adults, ${ }^{6}$ and a recent Cochrane review concluded that exercise has a statistically significant beneficial effect on balance in the short term, with the strength of the evidence being limited by the short duration of the studies. ${ }^{7}$ However, despite the evidence demonstrating the effectiveness of these 
interventions, their implementation in real-life settings has been challenging. Tai Chi requires a considerable amount of motivation and physical endurance. ${ }^{8}$ And in terms of adherence, exercise programs and vitamin D supplementation have shown variable and frequently discouraging results. ${ }^{7,9,10-12}$

Overall, due to the important role that balance disorders have in the pathogenesis of falls, ${ }^{3,4}$ and the limitations of the current interventions to improve balance in older fallers, ${ }^{7-12}$ the identification of new effective interventions is pivotal. In this study we aimed to evaluate the effect of a new virtualreality system (the Balance Rehabilitation Unit [BRU]) using virtual reality to assess balance and provide a training system for balance in a population of community-dwelling older subjects with a known history of falls.

\section{Methods}

\section{Subjects}

The study design is summarized in Figure 1. Participants (65 years and older) were recruited at the Falls and Fractures Clinic, Nepean Hospital in Penrith (NSW, Australia). Subjects attended the clinic after suffering at least one fall within 6 months prior to the assessment. All subjects were included in the study after showing poor performance in their balance, which was assessed using the accepted parameters of normality reported by the posturography component of the BRU. ${ }^{13-15}$ These parameters included limits of stability (LOS), interpreted as the maximum sway on the platform before losing stability, and area of the ellipse of confidence (CE) for the center of pressure (COP) at six different conditions: open eyes and eyes closed on hard surface, eyes closed on foam, and several different visual

Training group $(n=30)$

\begin{tabular}{|c|c|}
$\qquad \begin{array}{c}\text { 6 weeks } \\
\text { BRU-training } \\
20 \text { min twice a week }\end{array}$ & \\
& \\
\hline
\end{tabular}

\begin{tabular}{|l|} 
Control group $(\mathrm{n}=30)$ \\
U months
\end{tabular}

Figure I Study design.

Notes: Vertical lines indicate assessment points. Horizontal arrows indicate exposure to usual care with or without training. stimuli (saccadic condition combined with optokinetic bars in four different directions and visual-vestibular conditions) using the $3 \mathrm{D}$ virtual-reality goggles. Inclusion criteria were: LOS lower than $170 \mathrm{~cm}^{2}$ and COP $>20 \mathrm{~cm}^{2}$ in at least three of six different conditions. Exclusion criteria were: (a) severe visual impairment, (b) inability to ambulate independently with a cane or walker, (c) inability to stand unaided for 60 seconds, (d) score of $<22 / 30$ in the Mini-Mental State Examination, ${ }^{16}$ (e) Parkinson's disease or any neuromuscular conditions, (f) Geriatric Depression Scale $($ GDS $)>8 / 15,{ }^{17}$ and $(\mathrm{g})$ inability to understand or answer the study questionnaires. After agreeing to be part of the study and after informed consent was obtained, participants went through a comprehensive assessment that included structured questionnaires, information about chronic diseases, physical examination and anthropometric measurements. Subjects were then randomly assigned to either BRU-training or control groups. The study was conducted in accordance with the ethical standards set forth in the Declaration of Helsinki (1983). The local ethics committee at Nepean Blue Mountains Local Health District approved the project.

\section{Postural assessment}

BRU is a recently validated and reliable method that combines variable somatosensory, visual and vestibular conditions, which are used to assess and train balance. ${ }^{14,15,18-20}$ The assessment component of the BRU (posturography) evaluates postural control responses to different types of visual and visual-vestibular stimulation on standing surfaces of different firmness. The posturography report is automatically generated by the software integrated in the BRU system and includes LOS and COP under several different conditions. The assessment takes about 30 minutes to perform. To avoid any bias in the assessment, a physiotherapist performed all the posturography assessments at time 0 , at week 6 in the BRU-training group, and at month 9 in both groups. The same physiotherapist was responsible for asking the subjects about new falls and assisting them to perform the fear of falling questionnaires.

\section{Definition of falls}

Falls were defined as "an unexpected event in which the participants come to rest on the ground, floor, or lower level." ${ }^{21}$ The occurrence of falls was retrospectively assessed by asking the participant (1) whether they have suffered a fall and, (2) the number of falls during the 6 months prior to the assessment. 


\section{Gait assessment}

A GAIT Rite ${ }^{\circledR}$ (CIR Systems Inc, Havertown, PA, USA) instrumented walkway $(810 \mathrm{~cm} \times 89 \mathrm{~cm} \times 0.625 \mathrm{~cm}$, sample rate $=80 \mathrm{~Hz}$ ) was positioned along a straight section of the walkway to record spatiotemporal gait data.

\section{Grip strength}

Grip strength was measured following the Groningen Elderly Test using a Smedley Hand Dynamometer (Stoelting, Wood Dale, IL, USA). ${ }^{22}$ The best of three attempts (with 30 seconds rest between them) was recorded.

\section{Serum measurements}

Venous blood was collected from resting subjects for the measurement of serum 25(OH)D3, parathyroid hormone $(\mathrm{PTH})$, calcium, creatinine, and albumin. Serum 25(OH)D3 concentration was measured by chemiluminescence using the Elecsys 25(OH)D3 assay (Roche, Mannheim, Germany). Distributor is correct. The intra- and interassay precisions were $7.5 \%$ and $10.6 \%$, respectively (normal range $=10-132 \mathrm{nmol} / \mathrm{l}$ ). Intact PTH was measured by immunochemoluminometric assay (Immulite 2000 Siemens, Erlander, Germany; normal range $=12-72 \mathrm{pg} / \mathrm{mL}$ ). The intra- and interassay precisions were $7 \%$ and $5 \%$, respectively. Serum calcium, albumin, and creatinine were determined using automated standard laboratory methods. Because of the high prevalence of hypoalbuminemia in older adults, the serum concentration of albumin and calcium were used to correct the calcium value (calcium-corrected value $=\mathrm{Ca}+0.8$ [40-albumin]) The calcium-corrected value was used in the subsequent analysis. The clearance of creatinine was calculated from the Cockcroft formula ([(140 - age in years) $\times$ weight $(\mathrm{kg}) /$ $72 \times$ creatinine $\mathrm{mol} / \mathrm{L}])$. All measurements were performed at the Nepean Hospital, Penrith, Australia.

\section{Clinical assessment}

Depression was assessed using the Geriatric Depression Scale (GDS). Height was measured with a digital stadiometer. Nutritional assessment was performed by body mass index (BMI) calculation and by completing the Mini-Nutritional Assessment (MNA) tool.

\section{Fear of falling}

The Survey of Activities and Fear of Falling in the Elderly $(\mathrm{SAFFE})^{23}$ was used to assess fear of falling. To facilitate the analysis, a total SAFFE fear-of-falling score was generated as described previously. ${ }^{24}$ This method, which is based on a 5-point Likert ( $0-4$ item) response format with higher scores indicating a greater fear of falling, has demonstrated an internal consistency of $0.70 .{ }^{24}$

\section{BRU-training protocol}

The components of the BRU-training protocol are described in detail in Figure 2. Participants in the BRU-training group were scheduled for two sessions of balance training per week for 6 weeks under the guidance of a physiotherapist other than the one performing the assessments. Each training session lasted 30 minutes and consisted of a combination of visual-vestibular rehabilitation (Figure 2A) and postural training virtual reality exercises (Figure 2B). ${ }^{14}$ The rehabilitation exercises included saccadic, optokinetic stimulation, vestibular optokinetic and vestibular-ocular reflex exercises performed while standing. In addition, postural training virtual reality exercises were also used to improve balance (Figure 2B). These exercises consisted of three different postural training games with increasing levels of complexity (maximum of 15 levels), with the goal of reaching at least level 10 in every game by the end of the training period. The levels of complexity were progressively increased as the individual reported higher confidence and demonstrated learning of the correct postural control techniques required to pass to a higher level. After completing their 6 weeks of BRU training, our assessment physiotherapist obtained a new posturography report, which was then compared with the initial report (time 0).

\section{Falls prevention recommendations and interventions (usual care)}

After their initial assessment, all participants were given general recommendations and an evidence-based care plan on falls prevention (usual care). ${ }^{25}$ This usual care included an invitation to join an exercise program (following the Otago protocol), ${ }^{26}$ medication review, home visit by an occupational therapist (if more than $60 \%$ of the falls occurred at home), hearing and visual assessment, nutritional supplements (when nutritional risk was identified by the MNA tool), vitamin D supplementation (1,000 IU/day independently of their serum levels), and education materials on falls prevention.

\section{Sample size calculation}

The LOS measure was selected for calculation of sample size, based on our previous preliminary results. ${ }^{15}$ Expecting a posttraining improvement of $10 \%$, a sample size of 30 participants per group was required ( $80 \%$ power, alpha of 0.05$)$. This sample size was also considered sufficient when data from other balance measures (COP, CEs) were used. 



Figure 2 Components of the BRU-training protocol. Each training session lasted 30 minutes and consisted of a combination of visual-vestibular rehabilitation (A) and postural training virtual reality exercises $(\mathbf{B})$. The rehabilitation exercises $(\mathbf{A})$ included saccadic and optokinetic stimulation. Postural training exercises $(\mathbf{B})$ consisted of three different games (maze, breakfast and surfing) with increasing levels of complexity (maximum of 15 levels). ${ }^{33}$

\section{Statistical analysis}

Values are expressed as the mean of the standard deviations (SD). The baseline characteristics of the groups were compared using Student's unpaired $t$-test. We compared balance parameters within the BRU-training group at time 0 and after completing treatment ( 6 weeks) as well as between both groups at time 0 and 9 months using two-way analysis of repeated measures (ANOVA). When the ANOVA interaction was significant, means were compared by the NewmanKeuls method. Statistical significance was set at $P<0.05$. Statistics were performed using SPSS software (v. 20; IBM, Armonk, NY, USA).

\section{Results}

\section{Time 0: Before BRU training (both groups)}

Both groups showed similar demographic, anthropometric, and health characteristics at the beginning of the study. In addition, baseline balance and gait parameters were not significantly different between the two groups (Table 1).

\section{Week 6 post-BRU training}

\section{Adherence}

Of the 30 subjects in the BRU-training group, only two withdrew from the program before completing their 6 weeks of BRU training due to logistics problems to attend the sessions, which indicates a $97 \%$ adherence to the BRU-training program.

\section{Balance parameters}

Most of the participants (91\%) reached at least 10 out of 15 possible levels in every group of virtual exercises. We identified a significant improvement in balance parameters after 6 weeks of BRU training (Figure 3A). This improvement included a significant increase in $\operatorname{LOS}(\sim 31 \% ; P<0.01)$ and significantly smaller elliptical areas of the eyes closed on hard surface $(\sim-33 \% ; P<0.01)$, eyes closed on foam $(\sim-52 \%$; $P<0.01)$, optokinetic stimuli $(\sim 25 \% ; P<0.01)$, and vertical $(\sim 50 \% ; P<0.01)$ and horizontal $(\sim 33 \% ; P<0.01)$ VVC.

\section{Month 9: BRU-training and control groups}

Anthropometric, functional and biochemical characteristics of the participants were similar in both groups (data not shown). At month 9 post-initial assessments, both BRUtraining and control groups showed the same proportion of subjects attending an exercise program (2\%), taking nutritional supplements (3\%) and vitamin D supplementation (48\%), and reporting changes in their medications following the clinic's recommendations (11\%). As compared with the usual care group, the BRU-training group showed significantly higher level of LOS, and significantly smaller 
Table I Baseline characteristics of the subjects

\begin{tabular}{|c|c|c|}
\hline & $\begin{array}{l}\text { BRU training } \\
(n=30)\end{array}$ & $\begin{array}{l}\text { Control } \\
(n=40)\end{array}$ \\
\hline Age (years) & $79.3 \pm 10$ & $75 \pm 8$ \\
\hline Gender (\% women) & 63 & 61 \\
\hline Height (cm) & $160.5 \pm 6$ & $160.9 \pm 10$ \\
\hline BMI $\left(\mathrm{kg} / \mathrm{m}^{2}\right)$ & $27 \pm 6$ & $26 \pm 7$ \\
\hline Falls in the last year (\#) & $3.7 \pm 1.3$ & $3.8 \pm 2$ \\
\hline Vitamin D (nmol/L) & $68.3 \pm 18$ & $59 \pm 9$ \\
\hline Parathyroid hormone (pmol/L) & $6 \pm 5.4$ & $6 \pm 4$ \\
\hline Corrected calcium (mmol/L) & $2.3 \pm 0.2$ & $2.4 \pm 0.1$ \\
\hline Falls (last 6 months) & $3.5 \pm 4$ & $3.3 \pm 2$ \\
\hline Grip strength (kg) & $17 \pm 2$ & $20 \pm 3$ \\
\hline GDS (score/I5) & $4 \pm 2$ & $5 \pm 3$ \\
\hline SAFFE score & $2.14 \pm 0.63$ & $2.65 \pm 0.53$ \\
\hline Limits of stability $\left(\mathrm{cm}^{2}\right)$ & $|24 \pm 6|$ & $118 \pm 59$ \\
\hline $\begin{array}{l}\text { Eyes open on hard surface } \\
\left(\text { sway in } \mathrm{cm}^{2} \text { ) }\right.\end{array}$ & $5.8 \pm 3.3$ & $9.2 \pm 9$ \\
\hline $\begin{array}{l}\text { Eyes closed on hard surface } \\
\left.\text { (sway in } \mathrm{cm}^{2}\right)\end{array}$ & $11 \pm 9$ & $10 \pm 11$ \\
\hline $\begin{array}{l}\text { Eyes closed on foam } \\
\left(\text { sway in } \mathrm{cm}^{2}\right)\end{array}$ & $45 \pm 30$ & $36 \pm 26$ \\
\hline Optokinetic stimuli (sway in $\mathrm{cm}^{2}$ ) & $11 \pm 6$ & $9 \pm 3$ \\
\hline Vertical VVC (sway in $\mathrm{cm}^{2}$ ) & $19 \pm 15$ & $17 \pm 15$ \\
\hline Horizontal VVC (sway in $\mathrm{cm}^{2}$ ) & $17.1 \pm 11$ & $17 \pm 16$ \\
\hline Gait velocity $(\mathrm{cm} / \mathrm{sec})$ & $80 \pm 26$ & $75 \pm 25$ \\
\hline Cadence (steps/min) & $101 \pm 7$ & $97 \pm 21$ \\
\hline Stride length $(\mathrm{cm})$ & $91 \pm 18$ & $94 \pm 14$ \\
\hline Double support time (s) & $30 \pm 12$ & $29 \pm 10$ \\
\hline
\end{tabular}

Notes: Values are expressed as mean $\pm S D ; n=$ number of subjects. None of the parameters reached statistical significance.

Abbreviations: BMI, body mass index; BRU, balance rehabilitation unit; GDS, Geriatric Depression Scale; SAFFE, I/4 Survey of Activities and Fear of Falling in the Elderly; SD, standard deviation; VVC, visual-vestibular condition.

COP areas in the optokinetic stimuli and both vertical and horizontal VVC $(P<0.01)$. In contrast, elliptical areas for both eyes closed conditions (on hard surface and on foam) returned to the pre BRU-training levels.

\section{Falls and fear of falling}

As shown in Figure 3B, both groups showed a significant reduction in the incidence of falls $(P<0.05)$. However, BRU-training subjects reported a significantly lower number of falls as compared with the untrained controls $(1.1 \pm 0.7$ in the BRU-training vs $2 \pm 0.2$ in the control group; $P<0.01$ ). Finally, BRU-training subjects reported lower levels of fear of falling as demonstrated by a significantly lower SAFFE score in the BRU-training group compared with the control group (Figure 3C; $P<0.01$ ).

\section{Discussion}

In this study, we have assessed the effectiveness of a new virtual reality training system on balance, falls, and fear of falling in a population of community-dwelling older fallers.
Our system was expected to stress their balance and visiovestibular pathways while offering them a safe and closely supervised environment, which involves less physical effort than the usual balance exercise programs. An additional advantage of this system was a higher adherence that could be due to the level of physical effort involved and to the simplicity of the BRU-training protocol, which includes easy-to-follow instructions and a closer interaction between the health professional and the subject.

After completing their BRU-training protocol, participants showed a significant improvement in their LOS and COP. In addition, 9 months after their initial assessment, the BRU-training group showed a significant reduction in both the incidence of falls and in their levels of fear of falling. As expected, the subjects in the usual-care group showed a reduction in the number of falls; however, this reduction was significantly higher in the BRU-training group, which indicates an additive and beneficial effect of this intervention on fall prevention in this population. Interestingly, the positive effects of BRU training on balance seem to reduce with time as indicated by the results of our assessment at month 9 . Nevertheless, long-term studies looking at the maintenance of the beneficial effect of BRU training on these parameters are still required.

Although virtual reality has been used to rehabilitate subjects suffering from stroke, ${ }^{27}$ cerebral palsy, ${ }^{28}$ and brain injury, ${ }^{29}$ the use of this technology in falls prevention has been limited. Indeed, apart from the studies using the BRU in younger populations, ${ }^{18-20}$ there are no reports in the literature of the use of virtual reality to train balance and prevent falls in older subjects. The only related evidence came from a recent study that tested the Nintendo Wii ${ }^{\mathrm{TM}}$ Fit Plus in 26 young participants (aged $43 \pm 14$ years) suffering from balance impairment. ${ }^{30}$ Participants interacted with a television screen and no virtual reality was used. Although no data on change in balance parameters was provided, trained participants reported more enjoyment and motivation than usual physiotherapy, which coincides with the findings of the present study.

Our study has some strengths and limitations. The major strength of this study is that this is the first demonstration of the beneficial effect of balance training on balance parameters and falls in older adults using a novel, accurate, safe and effective virtual reality method. In terms of cost, with the exception of the initial investment on the BRU equipment and software, this intervention involves similar costing to any other falls prevention intervention that requires regular physiotherapy sessions. Another strength is the use of 
A

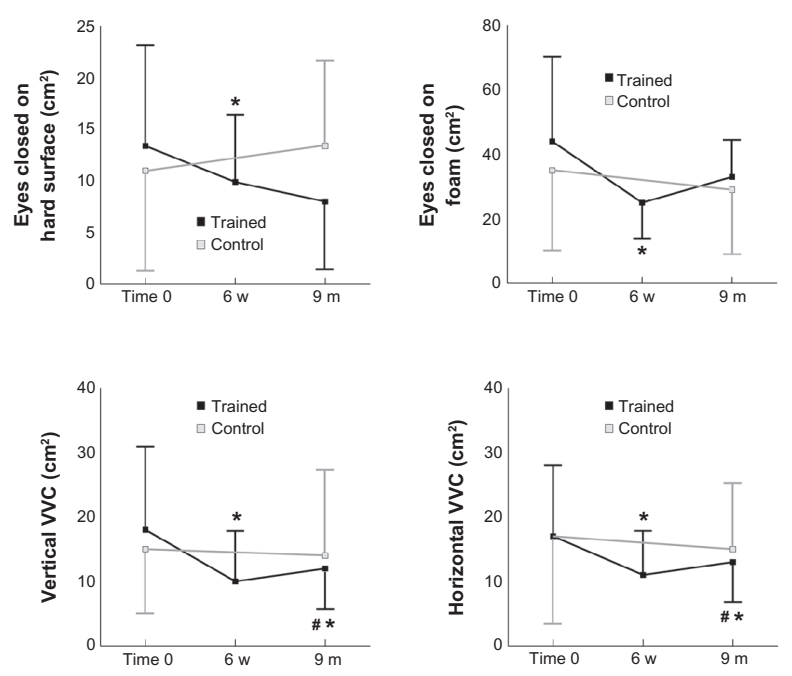

B

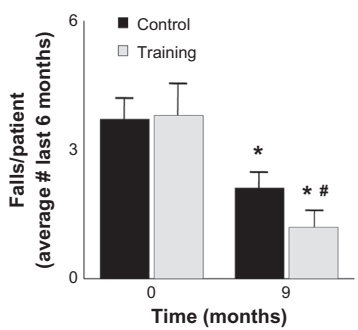

C

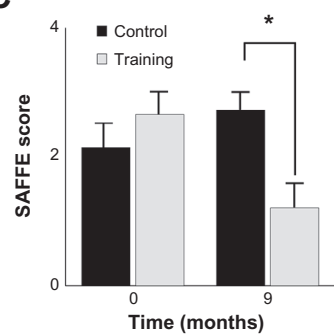

Figure 3 (A) Comparison of limits of stability and mean ellipse of confidence areas before and after 6 weeks of Balance Rehabilitation Unit (BRU) training (in the BRUtraining group) and after 9 months of the initial assessment in both BRU-training and control groups. (B and $\mathbf{C}$ ) Both groups showed a reduction in the number of falls at month 9 postassessment. BRU training induced a significant reduction in number of falls per subject (B) and fear of falling (C) in the BRU-training group compared with the untrained control group.

Notes: $* P<0.05$ significant intragroup difference from the baseline measure at 0 months; ${ }^{*} P<0.05$ intergroup difference after 9 months. A total SAFFE fear-of-falling score was generated based on a 5-point Likert (0-4) item response format, with higher scores indicating a greater fear of falling.

Abbreviations: BRU, Balance Rehabilitation Unit; SAFFE, Survey of Activities and Fear of Falling in the Elderly.

computerized posturography to document changes in balance parameters, which could be performed using the same BRU system. Although this method has the same sensitivity and specificity as the usual clinical balance tests, ${ }^{31,32}$ computerized posturography has major advantages such as a more precise determination of changes in balance parameters, no additional effort from the participant, and a more accurate inter- and intrarater reliability. ${ }^{31,32}$

In terms of the weaknesses of this study, performing a double-blind, controlled trial to test this system is clearly unfeasible, therefore we decided to use an open approach in which both the participants at the medical team were aware of the interventions. However, to prevent any assessment bias, different physiotherapists with no access to the subjects' data were specifically assigned to perform either assessment or training. Furthermore, the evidence that both groups are similar is provided in Table 1 and is demonstrated by similar involvement of the participants in our usual-care program.

Another potential limitation of the study is the use of self-reports for the falls data episodes, which could be inaccurate. However, excluding subjects with reported cognitive impairment could partially solve this limitation. Nevertheless, and although this study was not powered to assess its effect on falls prevention due to the small sample and the use of self-report, our finding that BRU training had a significantly higher effect on falls prevention deserves further exploration in future clinical trials.

A final limitation of this study could be that the control group was not exposed to the BRU equipment as often as the trained group, which could affect their performance at their second assessment. This is a very unlikely possibility since the posturography assessment, which is clearly different from the training component of BRU, is a very objective measurement that is independent of the subject's familiarity with the system.

In conclusion, we have successfully demonstrated that the novel BRU training is an effective method to offer balance training to community-dwelling older fallers. Although it is not intended to replace, but rather to complement, other falls prevention intervention programs, BRU training offers a safe and well-accepted intervention with high levels of effectiveness and adherence that could become a successful treatment for falls prevention in older adults in the near future.

\section{Acknowledgments}

This study was supported by a research grant from the Nepean Medical Research Foundation and by the Department of Geriatric Medicine at Nepean Hospital. Dr Demontiero holds a scholarship from The Rebecca Cooper Medical Research Foundation. 


\section{Disclosure}

The authors report no conflicts of interest in this work.

\section{References}

1. Close JC, Lord SR. Fall assessment in older people. BMJ. 2011; 343:d5153.

2. Nyman SR, Victor CR. Older people's recruitment, sustained participation, and adherence to falls prevention interventions in institutional settings: a supplement to the Cochrane systematic review. Age Ageing. 2011;40(4):430-436.

3. Wetmore SJ, Eibling DE, Goebel JA, et al. Challenges and opportunities in managing the dizzy older adult. Otolaryngol Head Neck Surg. 2011;144(5):651-656.

4. Rand D, Miller WC, Yiu J, et al. Interventions for addressing low balance confidence in older adults: a systematic review and meta-analysis. Age Ageing. 2011;40(3):297-306.

5. Muir SW, Montero-Odasso M. Effect of vitamin d supplementation on muscle strength, gait and balance in older adults: a systematic review and meta-analysis. J Am Geriatr Soc. 2011;59(12):2291-2300.

6. Liu H, Frank A. Tai chi as a balance improvement exercise for older adults: a systematic review. J Geriatr Phys Ther. 2010;33(3):103-109.

7. Howe TE, Rochester L, Jackson A, et al. Exercise for improving balance in older people. Cochrane Database Syst Rev. 2007;4:CD004963.

8. Wang C, Collet JP, Lau J. The effect of Tai Chi on health outcomes in patients with chronic conditions: a systematic review. Arch Intern Med. 2004;164(5):493-501.

9. Phillips EM, Schneider JC, Mercer GR. Motivating elders to initiate and maintain exercise. Arch Phys Med Rehabil. 2004;85(7 Suppl 3): $52-57$.

10. Sjösten NM, Salonoja M, Piirtola M, et al. A multifactorial fall prevention program in the community-dwelling aged: predictors of adherence. Eur J Public Health. 2007;17(4):464-470.

11. Tang BM, Eslick GD, Nowson C, et al. Use of calcium or calcium in combination with vitamin $\mathrm{D}$ supplementation to prevent fractures and bone loss in people aged 50 years and older: a meta-analysis. Lancet 2007;370(9588):657-662.

12. Grant AM, Avenell A, Campbell MK, et al. Oral vitamin D3 and calcium for secondary prevention of low-trauma fractures in elderly people (Randomised Evaluation of Calcium Or vitamin D, RECORD): a randomised placebo-controlled trial. Lancet. 2005;365(9471):1621-1628.

13. Baratto L, Morasso PG, Re C, Spada G. A new look at posturographic analysis in the clinical context: sway-density versus other parameterization techniques. Motor Control. 2002;6(3):246-270.

14. Suarez H, Geisinger D, Ferreira ED, et al. Balance in Parkinson's disease patients changing the visual input. Braz J Otorhinolaryngol. 2011;77(5):651-655.

15. Boersma D, Demontiero O, Mohtasham-Amiri Z, et al. Vitamin D status in relation to postural stability in the elderly. J Nutr Health Aging. 2012;16(3):270-275

16. Folstein MF, Folstein SE, McHugh PR. "Mini-mental state": A practical method for grading the cognitive state of patients for the clinician. J Psychiatr Res. 1975;12(3):189-198.
17. Wancata J, Alexandrowicz R, Marquart B, Weiss M, Friedrich F. The criterion validity of the Geriatric Depression Scale: a systematic review. Acta Psychiatr Scand. 2006;114(6):398-410.

18. Suarez H, Muse P, Suarez A, et al. Postural behavior responses to visual stimulation in patients with vestibular disorders. Acta Otolaryngol. 2000;120(2):168-172.

19. Suarez H, Geisinger D, Suarez A, et al. Postural control and sensory perception in patients with Parkinson's disease. Acta Otolaryngol. 2009;129(4):354-360.

20. Suárez H, Suárez A, Lavinsky L. Postural adaptation in elderly patients with instability and risk of falling after balance training using a virtualreality system. Int Tinnitus $J$. 2006;12(1):41-44.

21. Lamb SE, Jørstad-Stein EC, Hauer K, et al. Prevention of Falls Network Europe and Outcomes Consensus Group. Development of a common outcome data set for fall injury prevention trials: the Prevention of Falls Network Europe consensus. J Am Geriatr Soc. 2005;53(9): $1618-1622$.

22. Soer R, van der Schans CP, Geertzen JH, et al. Normative values for a functional capacity evaluation. Arch Phys Med Rehabil. 2009; 90(10):1785-1794.

23. Lachman ME, Howland J, Tennstedt S, et al. Fear of falling and activity restriction: the survey of activities and fear of falling in the elderly (SAFE). J Gerontol B Psychol Sci Soc Sci. 1998;53(1):P43-P50.

24. Li F, Fisher KJ, Harmer P, et al. Fear of falling in elderly persons: association with falls, functional ability, and quality of life. J Gerontol B Psychol Sci Soc Sci. 2003;58(5):P283-P290.

25. Micahel YL, Whitlock EP, Lin JS, et al. Primary care-relevant interventions to prevent falling in older adults: a systematic evidence review for the US Preventive Services Task Force. Ann Int Med. 2010;153(12):815-882.

26. Yang XJ, Hill K, Moore K, et al. Effectiveness of a targeted exercise intervention in reversing older people's mild balance dysfunction: a randomized controlled trial. Phys Ther. 2012;92(1):24-37.

27. Laver KE, George S, Thomas S, et al. Virtual reality for stroke rehabilitation. Cochrane Database Syst Rev. 2011;9:CD008349.

28. Brien M, Sveistrup H. An intensive virtual reality program improves functional balance and mobility of adolescents with cerebral palsy. Pediatr Phys Ther. 2011;23(3):258-266.

29. Gil-Gómez JA, Lloréns R, Alcañiz M, et al. Effectiveness of a Wii balance board-based system (eBaViR) for balance rehabilitation: a pilot randomized clinical trial in patients with acquired brain injury. J Neuroeng Rehabil. 2011;23:8-30.

30. Meldrum D, Glennon A, Herdman S, et al. Virtual reality rehabilitation of balance: assessment of the usability of the Nintendo Wii $\left({ }^{\circledR}\right)$ Fit Plus. Disabil Rehabil Assist Technol. 2012;7(3):205-210.

31. Browne J, O'Hare N. Development of a novel method for assessing balance: the quantitative posturography system. Physiol Meas. 2000; 21(4):525-534.

32. Browne J, O'Hare G, Finn A, Colin J. Clinical Assessment of the Quantitative Posturography System (QPS). Physiotherapy. 2002; 88(4):217-233.

33. http://www.medicaa.com/index2.php?option $=$ com_docman\&task $=$ doc view\&gid $=2 \&$ Itemid $=43$. Visited on: 11 February 2013
Clinical Interventions in Aging

\section{Publish your work in this journal}

Clinical Interventions in Aging is an international, peer-reviewed journal focusing on evidence-based reports on the value or lack thereof of treatments intended to prevent or delay the onset of maladaptive correlates of aging in human beings. This journal is indexed on PubMed Central, MedLine, the American Chemical Society's 'Chemical Abstracts Ser-

\section{Dovepress}

vice' (CAS), Scopus and the Elsevier Bibliographic databases. The manuscript management system is completely online and includes a very quick and fair peer-review system, which is all easy to use. Visit http://www.dovepress.com/testimonials.php to read real quotes from published authors. 